\title{
Editorial
}

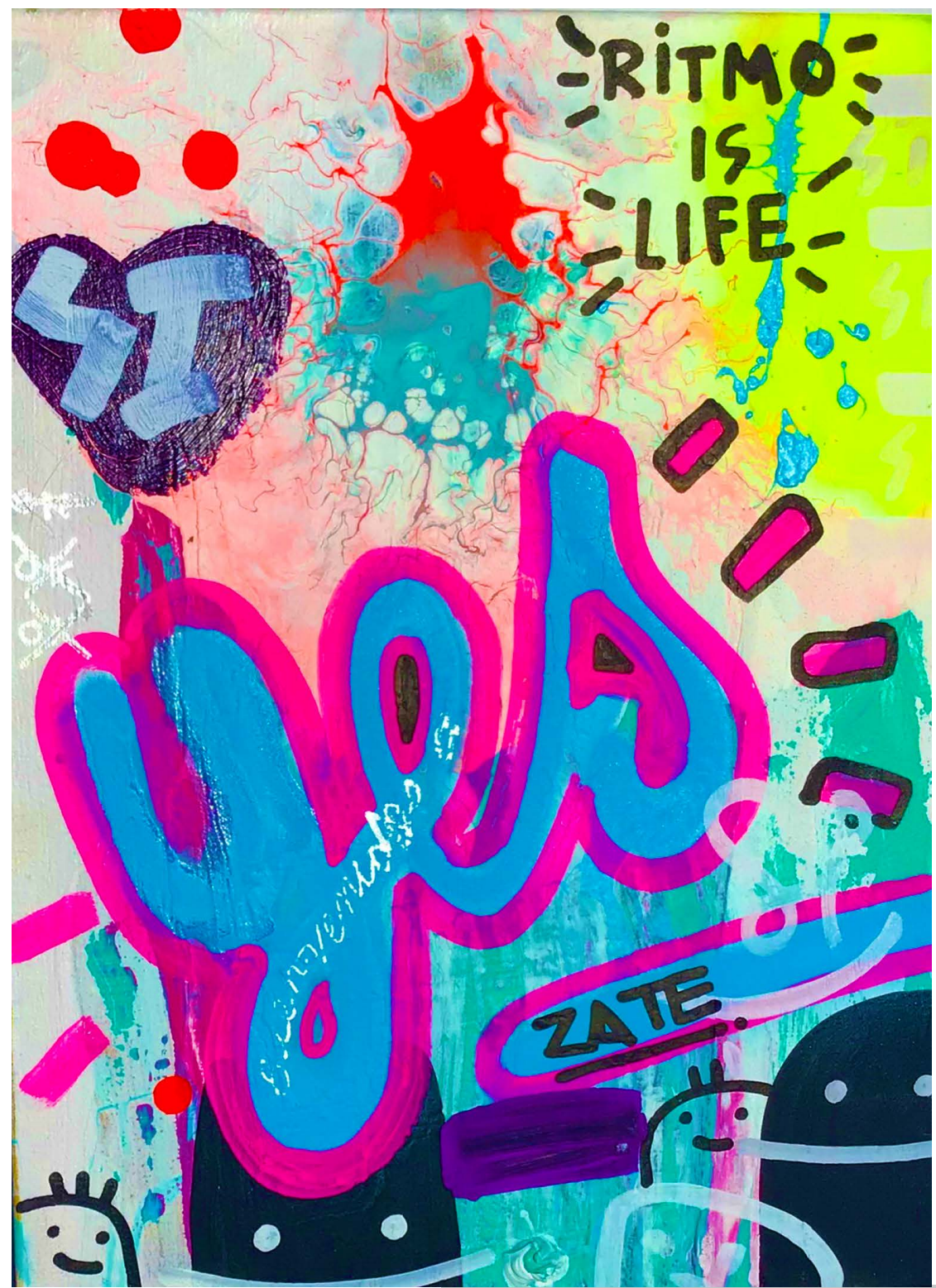

Artista invitado

Fabio Andrés Arboleda Mendoza

Yes

Técnica mixta sobre lienzo

$30 \times 35 \mathrm{~cm}$

2021

Medellín 


\section{El institucionalismo, el cuarto chispazo en la ciencia política*}

En 1996, cuando la Universidad de Oxford publicó A New Handbook of Polítical Science (Goodin y Klingemann, 2001), el institucionalismo como corriente de pensamiento en la ciencia política apenas estaba iniciando. Tan solo siete años antes James March y Johan Olsen (1997) publicaron su obra seminal El redescubrimiento de las instituciones. La base organizativa de la política. Tras esta embrionaria aparición, uno de los mayores historiadores de la disciplina, Gabriel Almond (2001), no nombró al institucionalismo en el segundo capítulo del manual, sino que advirtió sobre tres chispazos o momentos estelares en la "curva del progreso científico en el estudio de la política» en el siglo xx (p. 83): la Escuela de Chicago, entre 1920 y 1940; el conductismo, después de la Segunda Guerra Mundial; y «la entrada de los métodos deductivos y matemáticos y los modelo económicos del enfoque de la "elección racional-individualismo metodológico"» (p. 84) desde la década de 1970.

En ninguna parte de su clásico capítulo, Ciencia política: la historia de la disciplina, Almond (2001) alcanza a dimensionar lo que estaba pasando en esos momentos con el institucionalismo, ni a vislumbrar que muy pronto este enfoque se convertiría en otro giro revolucionario en la ciencia política, a pesar de afirmar en su obra previa, Una disciplina segmentada (Almond, 1999), que la ciencia política estaba abierta a las múltiples teorías, enfoques y metodologías que hacen más comprensible el mundo de la política. En aquel texto presenta un panorama histórico completo sobre los estudios políticos: inicia su recorrido con los griegos - desde Heródoto, Platón y Aristóteles-, luego pasa revista por los contractualistas clásicos -Hobbes, Locke y Rousseau- y los liberalistas y federalistas, hasta Ilegar,

\footnotetext{
* Texto derivado de los planteamientos realizados por el autor en la tesis doctoral en Estudios Políticos, Universidad Externado de Colombia, 2017, Organizarse para negociar la paz. Un análisis político transaccional de las estructuras de gobernanza de la paz negociada en Colombia, 1981-2016.
} 
finalmente, a la profesionalización de la ciencia política en el siglo xx, al referirse a la Escuela de Chicago, la revolución conductista de posguerra y la elección racional; incluso le queda tiempo para nombrar a las visiones posconductistas y antipluralistas, pero en ningún momento se detiene a analizar lo que se estaba cuajándose: el florecimiento de lo que se puede denominar actualmente por el mismo autor el cuarto chispazo en ciencia política.

Este texto presenta brevemente la evolución de lo que es el institucionalismo en la ciencia política, partiendo desde su inició hace tres décadas, para luego hacer una caracterización general del enfoque, su evolución y su estado actual. Con esto se intenta entregar algunos elementos que ayuden a actualizar la historia disciplinar de la ciencia política narrada por Almond (2001) hace un cuarto de siglo, la cual ha visto pasar por debajo mucha agua, logrando configurar al institucionalismo entre los principales enfoques de nuestra disciplina. Enfoques a los que, hasta hace una década, se les anteponía la denominación de los nuevos institucionalismos, pero dada su consolidación actual se omiten el plural y el mote de novedad, y se les reconoce como un aporte de similar magnitud al del conductismo o al de la teoría de la elección racional. Este enfoque ha permitido poner en diálogo a distintas disciplinas de las ciencias sociales, evidenciando el compromiso "con las reglas de la evidencia y la inferencia» de la política como ciencia» (p. 137).

\section{El contexto en el que surge el cuarto chispazo: el retorno a las instituciones}

La ciencia política es, tal vez, la más joven entre las ciencias sociales y humanas (Eslava, Orozco y Valencia, 2011). Su autonomía científica se logra construir tan solo a mediados del siglo xx. En aquel momento el dominio en los análisis politológicos lo detentaba el institucionalismo (Selznick, 1949; 1957), hoy denominado institucionalismo clásico. Este enfoque se esforzaba por estudiar las leyes formales, las reglas y las estructuras administrativas; sin embargo, para la naciente ciencia política este enfoque poco lograba explicar el comportamiento político real o sus resultados, de allí que la revolución conductista en la ciencia política — de la década de 1950 y principios de la de 1960 - aparezca como una reacción a este viejo institucionalismo (Shepsle, 1999). 
El conductismo buscaba la «explicación más allá de las estructuras formales» (Romero, 1999, p. 9), ponía la mirada en los deseos y comportamientos concretos y observables de los grupos e individuos. Algo similar pretendía, dos décadas después, la teoría de la elección racional. A partir de la tradición del individualismo metodológico, esta visión proveniente de las ciencias económicas intenta continuar la línea de trabajo del conductismo, haciendo énfasis en el individuo racional como agente político. Ambos enfoques tienen en común pensar a los actores políticos en el mismo nivel, haciendo parte de una estructura mecánica donde todos importan por igual para el funcionamiento del sistema político (Diz, Lois y Novo, 2012, p. 24).

Los conductistas realizaban un análisis que se caracterizaba por plantear análisis individuales atomizados y egoístas (March y Olsen, 1984 , p. 735). Argumentan que para entender la política y explicar sus resultados los análisis no se deben enfocar en los atributos formales de las instituciones gubernamentales, sino en la distribución informal del poder, en las actitudes y en el comportamiento político: «Es más, en contraste con lo que era percibido como un trabajo ateórico de académicos pertenecientes a una tradición formal-legal, el proyecto conductista como un todo era explícitamente teórico» (Thelen y Steinmo, 1992, p. 4). Así, el conductismo se caracteriza por observar los factores psicológicos, sociales, económicos y culturales que afectan el comportamiento político de los individuos, de ahí que se centre en el estudio de los comportamientos y actitudes de los individuos y los grupos en sí mismos para explicar los resultados políticos.

Por su parte, el enfoque de la elección racional pone el interés en la toma de decisiones políticas por parte de los individuos y colectividades, asumiendo una conducta racional y maximizadora de la utilidad de los actores políticos. Para este enfoque los agentes buscan maximizar su bienestar o del grupo al que representan, utilizando la información que tienen a su alcance. Realizan cálculos racionales que les permiten elegir, entre las diversas alternativas, la que mayor satisfacción produzca (Shepsle, 1986; Shepsle y Bonchek, 2005). Esta forma de analizar la política lleva al enfoque de la elección racional a buscar explicaciones de la conducta individual en el sistema de incentivos de los actores (Shepsle y Bonchek, 2005), a estudiar sus preferencias y su efecto en las decisiones (Hall y Taylor, 1996), y a describir las interacciones sociales y la forma como la decisión de un agente 
afecta las decisiones de otro. El modelo plantea que los actores políticos se comportan como agentes racionales maximizadores de su utilidad, donde la política es el resultado de esta conducta y de los equilibrios derivados de la selección que genera el predominio de aquellos actores.

En esta lógica de trabajo, tanto el conductismo como la elección racional examinan el comportamiento político como agregados de conductas individuales - subjetivo- (Marsh y Stoker, 1997), logrando construir una explicación coherente, aunque para muchos poco realista de la política (Thelen y Steinmo, 1992): «las bases teóricas de ambos enfoques anulan el espacio necesario para el estudio de las instituciones políticas como objeto de análisis: las instituciones desaparecen de la agenda de investigación principal de los enfoques preponderantes de la ciencia política» (Caballero, 2007, p. 10). Al finalizar el siglo xx, estos enfoques predominaban en la disciplina y sus explicaciones aún perduran, después de cinco décadas, en la ciencia política (Hay, 2002).

Pero ese olvido consciente del conductismo y de la elección racional de las instituciones tuvo una significativa consecuencia entre los críticos: sirvió para afianzar la idea de la necesidad de continuar con el estudio de este fenómeno. Para los estudiosos de la política, estos enfoques omitían «la importancia de la cancha y, por tanto, no podían responder a la cuestión crucial de por qué esos comportamientos y actitudes políticos y la distribución de recursos entre los grupos contendientes diferían de un país a otro» (Thelen y Steinmo, 1992, p. 5). Fijar la mirada solo en el «comportamiento de los actores sin analizar el entorno en el que actúan» es una gran debilidad, pues «en buena medida, determina tanto sus preferencias como los resultados de su acción» (Romero, 1999, p. 8).

De allí que, al finalizar la década de 1980, James March y Johan Olsen (1997) inauguran en la ciencia política una línea de trabajo que integra el análisis de la política con el estudio de las instituciones, conocida durante los primeros veinte años como los nuevos institucionalismos en la ciencia política (Caballero, 2007; Ortega, 2004; Basabe, 2007). Trabajo vector que diez años después llevó a Kenneth Shepsle (1999) a reconocer que el enfoque institucionalista era el dominante en la ciencia política, al menos en la estadounidense y británica (Eslava, Orozco y Valencia, 2011); es decir, en poco tiempo el institucionalismo - como nombre del enfoque general y 
en el que se agrupan todos los nuevos institucionalismos- se desarrolló y convirtió en uno de los paradigmas dominantes en la ciencia política.

En las dos últimas décadas del siglo $\mathrm{xx}$, se produjeron un conjunto de esfuerzos teóricos y aplicados que asumían la importancia de las instituciones políticas. Estos esfuerzos, que incorporan una variedad de enfoques y métodos, constituyen «el retorno de las instituciones» a la agenda de investigación principal de la ciencia política y constituyen la tradición que se ha dado en llamar «el nuevo institucionalismo» (Caballero, 2007, p. 10).

Se trata de un enfoque que retrotrae el interés de la política por los valores políticos, la cultura, los hábitos, las identidades y los propósitos colectivos, y que se basa en la idea de que las instituciones moldean y determinan la conducta de los agentes y, por tanto, las dinámicas de la política (Peters, 2003).

En conclusión, el institucionalismo en la ciencia política representa una crítica a la tradición conductista y de la elección racional dominante en esta ciencia desde su nacimiento (Eslava, Orozco y Valencia, 2011). Eso sí, retoman del conductismo la idea de los deseos y expectativas, pero introducen los hábitos y costumbres - tal como lo hace la vertiente sociológica del institucionalismo-; retoman la potencia analítica y predictiva del actor racional en la política, pero limitan su conducta y toma de decisiones por el ambiente institucional -idea desarrollada por el institucionalismo de la elección racional-; distingue entre las normas formales e informales del institucionalismo económico-; y reconoce las condiciones culturales e ideológicas - defendido por el institucionalismo histórico-. De esta manera, el institucionalismo en la ciencia política ha permitido, en un poco más tres décadas, ver con mayor claridad toda aquella rica dinámica que hay en la interacción social (Caballero, 2006, p. 195) en entornos inciertos, complejos y con presencia de instituciones — formales e informales-.

\section{La evolución de los estudios institucionales en la ciencia política y sus rasgos característicos}

Desde la aparición de los trabajos de March y Olsen $(1984 ; 1997)$ la investigación sobre instituciones políticas ha experimentado un continuo y notable desarrollo. El «retorno de las instituciones» a la agenda de 
investigación principal en ciencia política es, a principios del siglo xxı, una realidad generalmente aceptada, estudiando la noción, el papel y el cambio de las instituciones políticas (Caballero, 2007, p. 10). Esta rica dinámica evolutiva, de cerca de cuatro décadas, se puede dividir en dos grandes momentos: el primero inició con los escritos de March y Olsen desde mediados del decenio de 1980, y se extiende hasta iniciar el nuevo milenio, el cual se caracteriza por la aparición y competencia de una amplitud de enfoques y miradas instituciones; el segundo, que se viene presentando durante este nuevo siglo, se identifica con el diálogo multidisciplinar, el recogimiento y la integración de miradas en torno a las instituciones.

El primer momento de esta historia en la ciencia política comenzó tan pronto aparecieron los trabajos de March y Olsen (1984; 1997). A partir de allí se empezó a hablar de diferentes institucionalismos al servicio de esta. La acelerada producción de bibliografía alrededor de las instituciones (19842003) hizo notar la diversidad de enfoques que, de acuerdo a su tradición —sociológica, económica, jurídica e histórica, entre otras-, analiza las instituciones de manera particular, producción que llevó a reconocer en la ciencia política que no puede hablarse de un nuevo institucionalismo, sino de varios nuevos institucionalismos en esta ciencia (Peters, 2003), y que, como se advierte, abordan desde perspectivas distintas una variedad amplia de temas politológicos: «el nuevo institucionalismo no es una corriente de pensamiento homogénea; por el contrario, bajo este nombre se agrupa el trabajo académico realizado en diversas disciplinas de las ciencias sociales y desde enfoques teóricos relativamente diferentes» (Vergara, 1997, p. 17).

En orden de aparición, los trabajos más representativos alrededor de las instituciones en ciencia política son: James Buchanan (1984), Peter Evans, Dietrich Rueschemeyer y Theda Skocpol (1985), Kenneth Shepsle (1986), Elinor Ostrom (1986), Douglass North y Barry Weingast (1989), Douglas North (1990; 1993), Avinash Dixit (1990; 1996), Terry Moe (1990; 2005), Jon Elster (1991), Itai Sened (1991), Walter Powell y Paul Dimaggio (1999), Jack Knight (1992), Sven Steinmo, Kathleen Thelen y Frank Longstreth (1992), Arthur Denzau y Douglass North (1994), Peter Hall y Rosemary Taylor (1996), Barry Weingast (1995; 1996), Jack Knight e Itai Sened (1995), Guy Peters (1999/2003), Robert Goodin (1999), David Epstein y Sharyn O'Halloran (1999), Paul Pierson (2000), Jan-Erik Lane y Svante Ersson (2000), y Daniel Diermeier y Keith Krehbiel (2003). 
Esta riqueza bibliográfica llevó a que, al iniciar el siglo xxı, los analistas disciplinares identificaran y clasificaran los institucionalismos en varios enfoques. Aquí se presentan al menos tres de ellos: en primer lugar, Hall y Taylor (1996) identifican las vertientes histórica, de elección racional y sociológica; le sigue Peters (2003), que al finalizar el siglo hace un monumental trabajo sobre la escuela de los nuevos institucionalismos y divide en siete los enfoques: normativo, elección racional, histórico, empírico, sociológico, de representación de intereses e internacional; finalmente, Richard Scott (2001) los agrupa en regulatorio -económico-, normativo - ciencia política- y cognitivo —-sociológico-, y de forma similar lo hace Clara García (2001, p. 48) en histórico, neoclásico o institucionalismo - ligado al paradigma de la elección racional, económico y sociológico-.

La primera etapa del surgimiento de los nuevos institucionalismos permitió a la ciencia política ponerse en diálogo con otras ciencias hermanas como el derecho, la economía, la sociología, la historia y todas aquellas disciplinas donde los nuevos institucionalismos también habían comenzado a aparecer y posicionarse. Los nuevos institucionalismos permitieron establecer un diálogo, un acompañamiento y una complementación en el estudio de las instituciones. Así, al finalizar la primera década del siglo xxI, se logró tener en la ciencia política un mejorado conocimiento sobre las instituciones, en especial, de la gobernanza (Dixit, 2009); además de abordar temas clásicos de la política: gestión pública, burocracia estatal, grupos de presión, partidos políticos y desarrollo económico, político y social, usando para esto factores culturales, cognitivos, normativos y regulatorios que proporcionan estabilidad y sentido a la vida social (Scott, 2001, p. 48). En síntesis, se construyó un programa de investigación compartido que facilitó el reencuentro de la política con otras ciencias sociales, perdido cerca de un siglo atrás.

Precisamente, este reencuentro de la ciencia política con otras ciencias posibilitó que al iniciar el nuevo siglo se presentara un giro en el estudio de las instituciones que se ha mantenido hasta la actualidad. Entre los trabajos que siguen esta perspectiva integracionalista están: Oliver Williamson (2000; 2005; 2010), Richard Scott (2001), Masahiko Aoki (2001), Gonzalo Caballero (2002; 2006; 2007; 2011), Paul Joskow (2002), Victor Nee (2003), Robert Goodin (2003), Pablo Spiller y Mariano Tommasi (2003; 2005), Avner Greif y David Laitin (2004), Juan Manuel Ortega (2004), Terry Moe 
(2005), Thrainn Eggertsson (2005), Douglas North (2005a; 2005b), Kenneth Shepsle y Mark Bonchek (2005), Elinor Ostrom (2005; 2008), Gonzalo Caballero (2007), José Antonio Alonso y Carlos Garcimartín (2008), Sven Steinmo (2008/2013), Avinash Dixit (2009), Christopher Kingston y Gonzalo Caballero (2009), Mijael Altamirano y Abigail Martínez (2011), Dieter Nohlen (2011), Xóse Carlos Arias y Gonzalo Caballero (2013a; 2013b), Norman Schofield, Gonzalo Caballero y Daniel Kselman (2013), y Alexander González-Chavarría (2014).

Producción literaria que generó que desde los distintos nuevos institucionalismos —económico, elección racional, organizativo, legislativo, federativo, regulatorio, entre otros- se iniciara un nuevo ciclo de estudios institucionales, aquel que permitió el reencuentro, el diálogo y el trabajo conjunto. Complementariedad teórica que fue posible debido a tres componentes: en primer lugar, el compartir el mismo punto de partida, pues todos reconocen que «los arreglos institucionales y los procesos sociales son importantes» (Powell y DiMaggio, 1999, p. 36); segundo, el énfasis compartido en recuperar las instituciones como eje del análisis (Caballero, 2009) e insistir que «las instituciones condicionan la forma en la que las personas ven el mundo y no son meras reglas a las cuales se atienen» (Steinmo, 2013, p. 138), lo que permite hablar de una única denominación de institucionalismos; y tercero, una dinámica de trabajo que reconoce los avances que han tenido los diversos institucionalismos y que permiten construir un programa de investigación común, el cual recoge los logros conseguidos durante varias décadas de cada una de sus respectivas ciencias y que posibilita a la ciencia política analizar la estabilidad y el orden social, y entender los conflictos y cambios en las estructuras sociales.

Estos desarrollos más recientes de los institucionalismos introducen en la política el estudio de perspectivas novedosas como los derechos de propiedad, los costos de transacción, los diseños institucionales, los problemas de información y las estructuras y formas organizativas. A modo de ejemplo se encuentra el trabajo indagativo que realizó Oliver Williamson hasta su muerte el 21 de mayo de 2020 (Valencia, 2020). Este autor representa la punta del iceberg de una gran corriente de pensamiento sobre las instituciones, la cual ha contribuido en gran medida a la aparición del análisis político transaccional (Caballero, 2011; Arias y Caballero, 2013b; Valencia, 2020). Su propuesta teórica, aunque inicialmente buscaba su aplicación a la economía (Williamson, 1979; 1985; 1991), finalmente se 
extendió a otros ámbitos, entre ellos, a la ciencia política (Dixit, 1996; North, 1990; Williamson, 1999; 2000; 2005; 2010), concibiendo a las instituciones como estructuras de gobernanza, acuerdos sociales ajustados para reducir al mínimo los costos de transacción y que permitan interacciones más predictibles, confiables y eficientes (Williamson, 1985). En este sentido, «las instituciones constituyen soluciones relativamente eficientes a los problemas de la acción colectiva, pues las rutinas institucionales existen sobre todo para reducir los costos entre individuos y grupos con el objetivo de aumentar la eficiencia» (Romero, 1999, p. 13).

Williamson no continúa con la lógica de dividir los institucionalismos, sino de integrarlos, busca establecer un diálogo con los distintos enfoques, con el institucionalismo de la elección racional, con el histórico y con el económico, entre otros. Retoma del enfoque de la elección racional la idea de actores racionales y modelos subjetivos de análisis en la toma de decisiones políticas -individualismo metodológico-; del histórico la importancia del pasado en la comprensión de las ideologías, cuya idea se sintetiza en el concepto de senda o camino de la dependencia - path dependence o path dependency-, las cuales advierten de los cambios lentos que dan en las instituciones; $y$ finalmente, del nuevo institucionalismo económico la idea de diversos mecanismos de coordinación y la importancia de los costos y de la información en las transacciones de diverso tipo. (Valencia, 2020, p. 10).

De esta forma, a partir de su trabajo en 2001, Williamson hace un giro en su proyecto investigativo, proponiendo un diálogo multidisciplinar con el que buscó integrar enfoques, perspectivas de análisis y aportes variados, y en las dos décadas que le siguieron ayudó a construir un programa de investigación que reconoce los aportes de los nuevos institucionalismos en la ciencias sociales -economía, sociología, ciencia política, historia, psicología, entre otros-, logrando «construir y proponer una visión de conjunto; un programa que enfatiza en el matrimonio que hay entre la teoría política y las ciencias sociales, intentando proponer y construir un programa de investigación más completo, realista e integrador» (Valencia, 2020, p. 10). Con esto, el autor dejó para los estudios políticos «una agenda de investigación con una disparidad de intereses en variados temas que a hecho posible que surjan líneas de investigación abundantes (Dixit, 1996; North, 1990)» (p. 17) y ofreció una propuesta analítica que permite aplicar con toda su potencia al estudio de los fenómenos políticos. 
De similar manera, en la actualidad se encuentra un rico campo de investigación con autores como Elinor Ostrom (2005; 2008), Douglas North (2005a; 2005b) —que incluye a Chrysostomos Mantzavinos, Douglas North y Nathelie Shariq (2015) - y Sven Steinmo (2013), que han intentado realizar trabajos integradores similares a los de Williamson. Estos autores, desde temas distintos - la primera desde la gobernanza de bienes comunes, los segundos desde el desempeño de las instituciones en la sociedad y el tercero desde la orientación histórica y el papel de las instituciones-, defienden la importancia de las instituciones en la conducta de las personas y sus consecuencias, realización de intercambios políticos y el logro de los objetivos colectivos, y además presentan a las instituciones como susceptibles de diseño y rediseños —cambio institucional-, a pesar de ser producto también de la evolución social (North, 2005b), pensando a las instituciones como limitaciones ideadas por los hombres y mujeres para dar forma a la interacción humana y que estructuran incentivos en el intercambio políticos, por ejemplo, «en el seno de las burocracias o de las instituciones legislativas» (Caballero, 2007, p. 15).

\section{A modo de cierre}

En síntesis, al iniciar la tercera década del siglo xxı se ha logrado configurar un enfoque que ha revolucionado nuevamente a esta ciencia. Trabajo que se extiende a múltiples disciplinas y que viene siendo enriquecida por cada una de ellas.

La cuestión institucional ha recuperado protagonismo en el análisis político y económico durante los últimos años y diversos autores han incorporado las instituciones como parte central del examen de la realidad social; así, se ha comenzado a hablar de la existencia de una corriente contemporánea en las ciencias sociales, el nuevo institucionalismo. El redescubrimiento de las instituciones ha abierto una agenda interesante de investigación en política y economía comparadas (Romero, 1999, p. 7).

Una asombrosa variedad de enfoques de las instituciones ha permitido a la ciencia política un tratamiento de sus diversos problemas (Peters, 2003) y que con el pasar de los años han buscado integrarse para tener una visión más amplia y rica de la política. Esta tarea ha sido posible debido al reconocimiento colectivo de que las instituciones son el motor de la vida social, es decir, que tanto en las acciones individuales como las colectivas 
o grupales existen constreñimientos, normas, roles, costumbres y patrones de conducta que restringen, moldean o inciden en la toma de decisiones influyen en los deseos, preferencias y motivos- (Goodin, 2003, pp. 19-20).

Así, el institucionalismo en la ciencia política se presenta como una propuesta integradora, multidisciplinar y comprensiva de las instituciones, es un enfoque que rescata los clásicos estudios del Estado y la cultura, e intenta inyectar mayor realismo a la explicación y comprensión de un amplio espectro de fenómenos sociales (Evans, Rueschemeyer y Skocpol, 1985), el cual defiende a las instituciones como un rasgo estructural de la sociedad y como forma de gobernanza que permanece en el tiempo, afecta el comportamiento de los agentes y contribuye a las identidades políticas (Peters, 2003). Estos desarrollos han permitido a la ciencia política integrar los avances y aportes de los diversos institucionalismos, integrando categorías, variables e hipótesis a sus temas de estudio: «los nuevos institucionalismos muestran un camino de cómo avanzar hacia una integración interdisciplinar fundada en la acumulación de contribuciones teóricas y metodológicas» (Eslava, Orozco y Valencia, 2011, p. 24). Es un trabajo investigativocolaborativo que pone al servicio de los analistas de la política diversos enfoques teóricos y metodológicos, caracterizando a esta mirada como multidisciplinar, microanalítica y pragmática.

Germán Darío Valencia Agudelo (Colombia)**

\section{Cómo citar este artículo}

Valencia Agudelo, Germán Darío. (2021). El institucionalismo, el cuarto chispazo en la ciencia política. Estudios Políticos (Universidad de Antioquia), 62, pp. 9-25. https://doi.org/10.17533/udea.espo.n62a01

\section{Referencias bibliográficas}

1. Almond, Gabriel. (1999). Una disciplina segmentada. Escuelas y corrientes en la ciencia política. México, D. F.: Fondo de Cultura Económica.

\footnotetext{
** Director revista Estudios Políticos. Economista. Especialista en Gerencia Social. Magíster en Ciencia Política. Doctor en Estudios Políticos. Grupo Hegemonía, guerras y conflictos, Instituto de Estudios Políticos, Universidad de Antioquia UdeA. Calle 70 No. 52-21, Medellín, Colombia. Correo electrónico: german.valencia@udea.edu.co - Orcid: 0000-0002-6412-6986 - Google Scholar https:// scholar.google.es/citations?user=7Sm8z3MAAAAJ\&hl=es
} 
2. Almond, Gabriel. (2001). Ciencia política: la historia de la disciplina. En: Goodin, Robert y Klingemann, Hans-Dieter (eds.). Nuevo Manual de Ciencia Política. Tomo I (pp. 83-149). Madrid: Istmo.

3. Alonso, José Antonio y Garcimartín, Carlos. (2008). Acción colectiva y desarrollo. El papel de las instituciones. Madrid: Instituto Complutense de Estudios Internacionales.

4. Altamirano, Mijael y Martínez, Abigail. (2011). El método comparado y el Neoinstitucionalismo como marco metódologico para la investigación en las ciencias sociales. Mundo Siglo XXI, VII (25), pp. 55-63.

5. Aoki, Masahiko. (2001). Toward a Comparative Institutional Analysis. Cambridge: MIT. https://doi.org/10.7551/mitpress/6867.001.0001

6. Arias, Xóse Carlos y Caballero, Gonzalo. (2013a). Instituciones, costos de transacción y políticas públicas: un panorama. Revista de Economía Institucional, 5 (8), pp. 117-146.

7. Arias, Xóse Carlos y Caballero, Gonzalo. (eds.) (2013b). Nuevo institucionalismo: gobernanza, economía y políticas públicas. Madrid: Centro de Investigaciones Sociológicas.

8. Basabe, Santiago (comp.). (2007). El Nuevo Institucionalismo en Ciencia Política: perspectivas, enfoques y campos de acción. En: Instituciones e institucionalismo en América Latina (pp. 173-202). Quito: CIPEC.

9. Buchanan, James. (1984). Política sin romanticismos. Esbozo de una teoría positiva de la elección pública y de sus implicancias normativas. En: Buchanan, James; McCormick, Robert y Tollison, Robert (eds.). El análisis económico de lo político. Madrid: Instituto de Estudios Económicos.

10. Caballero, Gonzalo. (2002). El programa de la nueva economía institucional: lo macro, lo micro y lo político. Ekonomiaz, 50, pp. 230-261.

11. Caballero, Gonzalo. (2006). Abriendo la caja negra del Estado español: reglas, vetos, intereses y jerarquía en la formación de la política económica. Problemas del Desarrollo, 37 (144), pp. 192-219. https://doi.org/10.22201/ iiec.20078951e.2006.144.7603

12.Caballero, Gonzalo. (2007). Nuevo institucionalismo en ciencia política, institucionalismo de elección racional y análisis político de costes de transacción: una primera aproximación. Revista de Investigaciones Políticas y Sociológicas, 6 (2), pp. 9-27.

13. Caballero, Gonzalo. (2009). El papel de las instituciones en la geografía humana: un enfoque desde la nueva economía institucional. Economía, Sociedad y Territorio, ix (29), pp. 1-31.

14. Caballero, Gonzalo. (2011). Economía de las instituciones: de Coase y North a Williamson y Ostrom. Ekonomiaz, 77 (2), pp. 14-51. 
15. Denzau, Arthur \& North, Douglass. (1994). Shared Mental Models: Ideologies and Institutions. Kyklos, 47 (1), pp. 3-31. https://doi.org/10.1111/j.1467-6435.1994. tb02246.x

16. Diermeier, Daniel \& Krehbiel, Keith. (2003). Institutionalism as a Methodology. Journal of Theoretical Politics, 15 (2), pp. 123-144. https://doi. org/10.1177/0951629803015002645

17. Dixit, Avinash. (1990). A Transaction Cost Theory of Politics. Journal of TheoreticalPolitics, 2(4),pp.355-367.https://doi.org/10.1177/0951692890002004001

18. Dixit, Avinash. (1996). The Making of Economic Policy: A Transaction-Cost Politics Perspective. Boston: MIT. https://doi.org/10.7551/mitpress/4391.001.0001

19. Dixit, Avinash. (2009). Governance Institutions and Economic Activity. American Economic Review, 99 (1), pp. 3-24. https://doi.org/10.1257/aer.99.1.5

20. Diz, Isabel; Lois, Marta y Novo, Amparo. (2012). Ciencia política contamporanea. Barcelona: UOC.

21. Eggertsson, Thrainn. (2005). Imperfect Institutions. Opportunities and Limits of Reform. University of Michigan. https://doi.org/10.3998/mpub.91126

22. Elster, Jon. (1991). El cemento de la sociedad: las paradojas del orden social. Barcelona: Gedisa.

23. Eslava, Adolfo, Orozco, Hernán y Valencia, Germán. (2011). Los nuevos institucionalismos como riqueza metodológica para el estudio de la política. Anuario Opera, 11, pp. 5-28. https://revistas.uexternado.edu.co/index.php/opera/article/ view/3561

24. Epstein, David \& O'holloran, Sharyn. (1999). Delegating Powers. A Transaction Cost Politics Approach to Policy Making Under Separate Powers. Cambridge: Cambridge University. https://doi.org/10.1017/CBO9780511609312

25. Evans, Peter; Rueschemeyer, Dietrich \& Skocpol, Theda. (1985). Bringing the State Back In. Cambridge: Cambridge University. https://doi.org/10.1017/ CBO9780511628283

26. García, Clara Eugenia. (2001). Análisis económico de las organizaciones. Enfoques y perspectivas. Madrid: Alianza.

27. González-Chavarría, Alexander. (2014). Análisis institucional multiagente: el problema de estructuración y agencia en la explicación de la emergencia de estructuras de gobernanza. Revista de Estudios Sociales, 49, pp. 190-204. https://doi. org/10.7440/res49.2014.14

28. Goodin, Robert. (1999). Rationality Redux: Reflections on Herbert A. Simon's vision of Politics. In: Alt, James; Levi, Margaret y Ostrom, Elinor (Eds.). Competition and Cooperation (pp. 60-84). New York: Russell Sage Foundation.

29. Goodin, Robert. (2003). Teoria del diseño institucional. Barcelona: Gedisa.

30. Goodin, Robert \& Klingemann, Hans-Dieter. (2001). A New Handbook of Political Science. New York: Oxford University. 
31. Greif, Avner \& Laitin, David. (2004). A Theory of Endogenous Institutional Change. American Political Science Review, 98 (4), pp. 633-652. https://doi. org/10.1017/S0003055404041395

32. Hall, Peter \& Taylor, Rosemary. (1996). Political Science and the Three New Institutionalisms. Political Studies, xuIv, pp. 936-957. https://doi. org/10.1111/j.1467-9248.1996.tb00343.x

33. Hay, Colin. (2002). Political Analysis. London: Palgrave. https://doi. org/10.1007/978-0-230-62911-0

34. Joskow, Paul. (2002). Transaction Cost Economics, Antritust Rules and Remedies. Journal of Law, Economics and Organization, 18, pp. 95-116. https://doi. org/10.1093/jleo/18.1.95

35.Kingston, C Christopher \& Caballero, Gonzalo. (2009). Comparing Theories of Institutional Change. Journal of Institutional Economics, 5 (2), pp. 151-180. https:// doi.org/10.1017/S1744137409001283

36. Knight, Jack. (1992). Institutions and Social Conflict. Cambridge: Cambridge University. https://doi.org/10.1017/CBO9780511528170

37. Knight, Jack \& Sened, Itai. (eds.). (1995). Explaining Social Institutions. Michigan: University of Michigan. https://doi.org/10.3998/mpub.14827

38.Lane,Jan-Erik\&Ersson,Svante.(2000). TheNewInstitutionalPolitics. Performance and outcomes. London: Routledge. https://doi.org/10.4324/9780203264607

39. Mantzavinos, Chrysostomos; North, Douglas y Shariq, Nathalie. (2015). Aprendizaje, instituciones y desempeño económico. Revista Economía y Región, 9 (1), pp. 11-34. https://revistas.utb.edu.co/index.php/economiayregion/article/view/94

40. March, James \& Olsen, Johan. (1984). The New Institutionalism: Organizational Factors in Political Life. American Political Science Review, 78, pp. 734-749. https:// doi.org/10.2307/1961840

41. March, James y Olsen, Johan. (1997). El redescubrimiento de las instituciones. La base organizativa de la política. México, D. F.: Universidad Autónoma de Sinaloa y Fondo de Cultura Económica.

42. Marsh, David y Stoker, Gerry. (1997). Teoría y métodos de la Ciencia Política. Madrid: Alianza.

43. Moe, Terry. (1990). Political Institutions: The Neglected Side of the Story. Journal of Law, Economics and Organization, 6, pp. 213-253. https://doi.org/10.1093/ jleo/6.special_issue.213

44. Moe, Terry. (2005). Power and Political Institutions. Perspectives on Politics, 3 (2), pp. 215-233. https://doi.org/10.1017/S1537592705050176

45. Nee, Victor. (2003). New Institutionalism, Economic and Sociological. Center for the study of Economy \& Society. Working Paper Series, 4. http://www.soc.cornell. edu/faculty/nee/pubs/newinstitutionalism.pdf

46. North, Douglass. (1990). A Transaction Cost Theory of Politics. Journal Theorical Politics, 2 (4), pp. 355-367. https://doi.org/10.1177/0951692890002004001 
47. North, Douglas. (1993). Instituciones, cambio institucional y desempeño económico. México, D. F.: Fondo de Cultura Económica.

48. North, Douglass. (2005a). Institutions And the Performance of Economies Over Time. In: Ménard, Claude \& Shirley, Mary (Eds.). Handbook of New Institutional Economics (pp. 1-16). Cheltenham: Edward Elgar.

49. North, Douglass. (2005b). Understanding the Process of Economic Change. Princeton: Princeton University.

50. North, Douglass \& Weingast, Barry. (1989). Constitutions and Commitment: The Evolution of Institutions Governing Public Choice in Seventeenth- Century England. The Journal of Economic History, 49 (4), pp. 803-832. https://doi.org/10.1017/ S0022050700009451

51. Nohlen, Dieter. (2011). La democracia, instituciones, conceptos y contexto. México D. F.: UNAM.

52. Ortega, Juan Manuel. (2004). El nuevo institucionalismo en la ciencia política. Revista Mexicana de Sociología, 99, pp. 49-57. https://doi.org/10.2307/3541442

53. Ostrom, Elinor. (1986). An Agenda for the Study of Institutions. Public Choice, 48, pp. 3-25. https://doi.org/10.1007/BF00239556

54. Ostrom, Elinor. (2005). Understanding Institucional Diversity. Princeton: Princeton University.

55. Ostrom, Elinor. (2008). Institutions and the Environment. Economic Affairs, 28 (3), pp. 24-31. https://doi.org/10.1111/j.1468-0270.2008.00840.x

56. Peters, Guy. (2003). El nuevo institucionalismo. Teoría institucional en la ciencia política. Barcelona: Gedisa.

57. Pierson, Paul. (2000). Increasing Returns, Path Dependence, and the Study of Politics. American Political Science Review, 94 (2), pp. 251-267. https://doi. org/10.2307/2586011

58. Powell, Walter y Di Maggio, Paul (eds.). (1999). El nuevo institucionalismo en el análisis organizacional. México: UAEM y Fondo de Cultura Económica.

59. Romero, Jorge. (1999). Estudio introductorio. Los nuevos institucionalismos: sus diferencias, sus cercanías. En: Powell, Walter y DiMaggio, Paul (eds.). El nuevo institucionalismo en el análisis organizacional (pp. 7-29). México, D. F.: UAEM y Fondo de Cultura Económica.

60. Schofield, Norman; Caballero, Gonzalo y Kselman, Daniel. (2013). Advances in Political Economy: Institutions, Modelling and Empirical Analysis. Berlin: Springer.

61. Scott, W. Richard. (2001). Institutions and Organizations. Thousand Oaks: Sage.

62. Selznick, Philip. (1949). TVA and the Grass Roots. Berkeley: University of California.

63. Selznick, Philip. (1957). Leadership in Administration. Evanston: Row, Peterson. 
64. Shepsle, Kenneth. (1986). Institutional Equilibrium and Equilibrium Institutions. In: Weisburg, Herbert (Comp.). Political Science: The Science of Politics (pp. 51-82). New York: Agathon.

65. Shepsle, Kenneth. (1999). El estudio de las instituciones: lecciones del enfoque de la elección racional. En: Farr, James; Dryzek, John y Stephen, Leonard (eds.). La ciencia política en la historia (pp. 354-377). Madrid: Istmo.

66. Shepsle, Kenneth y Bonchek, Mark. (2005). Las fórmulas de la política. Instituciones, racionalidad y comportamiento. México, D. F.: CIDE-Taurus-Santillana.

67. Sened, Itai. (1991). Contemporary Theory of Institutions in Perspective. Journal of Theoretical Politics, 3 (4), pp. 379-402. https://doi. org/10.1177/0951692891003004002

68. Spiller, Pablo \& Tommasi, Mariano. (2003). The Institutional Foundations of Public Policy: a Transactions Approach with Application to Argentina. Journal of Law Economics and Organization, 19 (2), pp. 281-306. https://doi.org/10.1093/jleo/ ewg012

69. Spiller, Pablo \& Tommasi, Mariano. (2005). The Institutions of Regulations: An application to public utilities. In: Menard, Claude y Shirley, Mary (Eds.). Handbook of New Institutional Economics (pp. 515-543). Cheltenham: Edward Elgar. https://doi. org/10.1007/978-3-540-69305-5_21

70. Steinmo, Sven. (2013). Institucionalismo histórico. En: Della Porta, Donatella y Michael Keating (eds.). Enfoques y metodologías de las ciencias sociales (pp. 131152). Madrid: Akal.

71. Steinmo, Sven; Thelen, Kathleen \& Longstreth, Frank. (1992). Structuring Politics: Historical Institutionalism in Comparative Analysis. Cambridge: Cambridge University. https://doi.org/10.1017/CBO9780511528125

72. Thelen, Kathleen \& Steinmo, Sven. (1992). Historical Institutionalism in Comparative Politics. In: Steinmo, Sven; Thelen, Kathleen y Longstreth, Frank (Eds.). Structuring Politics. Historical Institutionalism in Comparative Analysis (pp. 1-33). Cambridge: Cambridge University. https://doi.org/10.1017/CBO9780511528125.002

73. Valencia Agudelo, Germán Darío. (2020). Editorial. El análisis político transaccional de Oliver Williamson. In memoriam. Estudios Políticos (Universidad de Antioquia), 59, pp. 9-22. https://doi.org/10.17533/udea.espo.n59a01

74. Vergara, Rodolfo. (1997). «El redescubrimiento de las instituciones»: de la teoría organizacional a la ciencia política (pp. 9-40). En: March, James y Olsen, Johan. El redescubrimiento de las instituciones. La base organizativa de la política. México, D. F.: Universidad Autónoma de Sinaloa y Fondo de Cultura Económica.

75. Weingast, Barry. (1995). The Economic Role of Political Institutions: MarketPreserving Federalism and Economic Development. The Journal of Law, Economics and Organization, 11 (1), pp. 1-31.

76. Weingast, Barry. (1996). Political Institutions: Rational Choice Perspectives. In: Goodin, Robert \& Klingemann, Hans-Dieter (Eds.). A New Handbook 
of Political Science (pp. 167-190). Oxford: Oxford University. https://doi. org/10.1093/0198294719.003.0005

77. Williamson, Oliver. (1979). Transaction-Cost Economics: The Governance of Contractual Relations. The Journal of Law and Economics, 22 (2), pp. 233-261. https:// doi.org/10.1086/466942

78. Williamson, Oliver. (1985). Reflections on the New Institutional Economics. Journal of Institutional and Theoretical Economics, 141 (1), pp. 187-95.

79. Williamson, Oliver. (1991). Comparative Economic Organization: The Analysis of Discrete Structural Alternatives. Administrative Science Quarterly, 36 (2), pp. 269-296. https://doi.org/10.2307/2393356

80. Williamson, Oliver. (1999). Public and Private Bureaucracies: A Transaction Cost Economics Perspective. The Journal of Law, Economics, \& Organization, 15 (1), pp. 306-342. https://doi.org/10.1093/jleo/15.1.306

81. Williamson, Oliver. (2000). The New Institutional Economics: Taking Stock, Looking Ahead. Journal of Economic Literature, 38 (3), pp. 595-613. https://doi. org/10.1257/jel.38.3.595

82. Williamson, Oliver. (2005). The Economics of Governance. The American Economic Review, 95 (2), pp. 1-18. https://doi.org/10.1257/000282805774669880

83. Williamson, Oliver. (2010). Transaction Cost Economics: The Natural Progression. The American Economic Review, 100 (3), pp. 673-690. https://doi. org/10.1257/aer.100.3.673 